\title{
Erratum to: Psychosis in Parkinson's Disease: Epidemiology, Pathophysiology, and Management
}

\author{
Anna Chang ${ }^{1,2} \cdot$ Susan H. Fox ${ }^{1}$
}

Published online: 8 August 2016

(c) Springer International Publishing Switzerland 2016

\section{Erratum to: Drugs (2016) 76:1093-1118 DOI 10.1007/s40265-016-0600-5}

\section{Page 1093, Key Points box,}

The last point, which previously read:

Newer agents are in development but comparative efficacy and safety is as yet unknown.

Should read:

Pimavanserin was approved recently in the USA and newer agents are in development; however, comparative efficacy and safety are as yet unknown.

Page 1107, Table 5, entitled 'Drugs used to treat PD psychosis'

The dosage for pimavanserin, which previously read:

$40 \mathrm{mg} /$ day

Should read:

$34 \mathrm{mg} /$ day (equivalent to pimavanserin tartrate $40 \mathrm{mg} /$ day)

The online version of the original article can be found under doi:10.1007/s40265-016-0600-5.

Susan H. Fox

sfox@uhnresearch.ca

1 Morton and Gloria Shulman Movement Disorder Clinic, University of Toronto, Toronto Western Hospital, 7th Floor, McLaughlin Pavilion, 399 Bathurst Street, Toronto, ON M5T 2S8, Canada

2 Department of Neurology, Shin Kong Wu Ho-Su Memorial Hospital, Taipei, Taiwan
The side effects for pimavanserin, which previously read: Sedation; postural hypotension

Should read:

Oedema; confusional state

Page 1108, Section 4.1.3, paragraph 3

Sentence 5, which previously read:

A phase II study in 46 patients reported good tolerability over 28 days with a small reduction in psychosis versus placebo using SAPS.

Should read:

A phase II study in 59 patients reported good tolerability over 28 days with a small reduction in psychosis versus placebo using SAPS.

Last sentence, which previously read:

Eleven percent of patients in the treatment group withdrew from the trial because of worsening psychotic symptoms.

Should read:

Six patients in the treatment group withdrew from the trial because of worsening psychotic symptoms. 